\title{
Existence of integro-differential solutions for a class of abstract partial impulsive differential equations
}

\author{
Marcos N Rabelo ${ }^{1 *}$, Marcos Henrique ${ }^{2}$ and Giovana Siracusa ${ }^{1}$
}

\author{
* Correspondence: rabelo@dmat. \\ ufpe.br \\ 1 Departamento De Matemática, \\ Universidade Federal De \\ Pernambuco, Recife-pe, Cep 50540- \\ 740, Brazil \\ Full list of author information is \\ available at the end of the article
}

\begin{abstract}
In this study, we investigate the existence of integro-differential solutions for a class of abstract partial impulsive differential equations.

Keywords: integro-differential equations, neutral differential equations, analytic semigroup of compact operators; non-autonomous operators, family of evolution operators, mild solutions
\end{abstract}

\section{Introduction}

In this study, we established two existence results of solutions for a class of impulsive functional differential equations which can be described in the following form

$$
\begin{aligned}
& \frac{\mathrm{d}}{\mathrm{d} t} D\left(t, u_{t}\right)=A(t) D\left(t, u_{t}\right)+f\left(t, u_{t}, \int_{0}^{t} e\left(t, s, u_{s}\right) \mathrm{d} s\right), t \in[0, b], t \neq t_{i}, \\
& u_{0}=\phi, \phi \in \mathcal{B}, \\
& \Delta u\left(t_{i}\right)=I_{i}\left(u_{t_{i}}\right), \quad i \in\{1, \ldots, m\}
\end{aligned}
$$

where $A(t): D(A(t)) \subset \mathbb{X} \rightarrow \mathbb{X}$ is a family of unbounded linear closed operators such that for each $t \in[0, b], A(t)$ is the infinitesimal generator of analytic semigroup of linear bounded operators $\left(S_{t}(s)\right)_{s \geq 0}$ on a Banach space $\mathfrak{X}$, endowed with the norm $\|\cdot\|_{\mathfrak{X}}$; the history $u_{t}:(-\infty, 0] \rightarrow \mathbb{X}$ is defined as $u_{t}(\theta)=u(t+\theta), \theta \leq 0 ; \mathcal{B}$ is a set of measurable functions $\phi:(-\infty, 0] \rightarrow X$ endowed with appropriate seminorm; the operator $D(t, \varphi)$ is defined as $D(t, \varphi)=\varphi(0)+g(t, \varphi)$, where the functions $f:[0, b] \times \mathcal{B} \times \mathbb{X} \rightarrow \mathbb{X}, f:[0, b] \times \mathcal{B} \times \mathbb{X} \rightarrow \mathbb{X}$ and $I_{i}: \mathbb{X} \rightarrow \mathbb{X}, i \in \mathbb{Z}$ are appropriate functions for all $i \in\{1, \ldots, m\} ; 0<t_{1}<\ldots<t_{m}<b$ is a sequence of fixed real numbers and the symbol $\Delta \xi(t)$ represents the jump of the function $\xi$ at the moment $t$, this means that $\Delta \xi(t)=\xi\left(t^{+}\right)-\xi\left(t^{-}\right)$, where the notation $\xi\left(t^{+}\right)$and $\xi\left(t^{-}\right)$represent, respectively, the right and the left-hand side limits of the function $\xi$ at $t$.

There are many physical phenomena that are described by means of impulsive differential equations, for instance, biological systems, electrical engineering, chemical reactions, among others can be modeled by impulsive differential equations, a good survey on impulsive differential equations can be found in [1] see also [2,3]. However,

\section{Springer}

C 2011 Rabelo et al; licensee Springer. This is an Open Access article distributed under the terms of the Creative Commons Attribution License (http://creativecommons.org/licenses/by/2.0), which permits unrestricted use, distribution, and reproduction in any medium, provided the original work is properly cited. 
impulsive actions can influence the behavior of solutions making the analysis more difficult. Motivated by this facts, the studies of such systems have drawn the attention of many researchers during last years.

Recently, Park et al. [4] have investigated the problem

$$
\begin{gathered}
\frac{\mathrm{d}}{\mathrm{d} t}\left[x(t)-g\left(t, x_{t}, \int_{0}^{t} a\left(t, s, x_{s}\right) \mathrm{d} s\right)\right]=A x(t)+f\left(t, x_{t}, \int_{0}^{t} e\left(t, s, x_{s}\right) \mathrm{d} s\right), \\
t \in[0, b], t \neq t_{i}, i=1,2, \ldots, m, \\
\Delta x\left(t_{i}\right)=I_{i}\left(x\left(t_{i}^{-}\right)\right), \quad i=1,2, \ldots, m, \\
x_{0}=\phi \in \mathcal{B}_{h} .
\end{gathered}
$$

In this model, the operator $A: D(A) \subset \mathbb{X} \rightarrow \mathbb{X}$ is the infinitesimal generator of a compact analytic semigroup of bounded linear operators $(T(t))_{t \geq 0}$ on a Banach space X such that $0 \in \rho(A)$, where $\rho(A)$ is the resolvent set of the operator $f, g:[0, b] \times \mathcal{B}_{h} \times \mathbb{X} \rightarrow \mathbb{X}, f, g:[0, b] \times \mathcal{B}_{h} \times \mathbb{X} \rightarrow \mathbb{X}$ and $I_{i}: \mathbb{X} \rightarrow \mathbb{X}, i=1,2, \ldots, m$ are given functions that satisfy suitable conditions. Using the theory of fractional powers and priori estimates for compact operators, the authors established some existence result for the problem (1.4). Lately, Balachandran and Annapoorani [5] investigated the following class of abstract problem (1.5)

$$
\begin{aligned}
\frac{\mathrm{d}}{d t}\left[x(t)-g\left(t, x_{t}\right)\right] & =A(t) x(t)+f\left(t, x_{t}\right)+\int_{0}^{t} e\left(t, s, x_{s}\right) \mathrm{d} s, t \in I=[0, b], t \neq t_{i}, i=1,2, \ldots, m, \\
\Delta x\left(t_{i}\right) & =I_{i}\left(x\left(t_{i}^{-}\right)\right), \quad i=1,2, \ldots, m, \\
x_{0} & =\phi \in \mathcal{B}_{h} .
\end{aligned}
$$

In the system (1.5), it was assumed that for each $t \in[0, b]$ the operator $A(t)$ is the infinitesimal generator of compact analytic semigroup of bounded linear operators on a Banach space $\mathfrak{X}$. Moreover, the domain, $D(A(t))$, of the operators $A(t)$ is assumed to be independent of $t \in[0, b]$ and dense in $\mathbb{X}$, i.e., $A(t): D \subset \mathbb{X} \rightarrow \mathbb{X}$ with $\bar{D}=\mathbb{X}$. To get their results, the authors used the conditions of Acquistapace and Terreni, see [6], to guarantee the existence of an evolution family of operators associated with the nonautonomous abstract Cauchy problem

$$
\begin{aligned}
& u^{\prime}(t)=A(t) u(t), t \in[0, b], \\
& u(0)=x \in \mathbb{X} .
\end{aligned}
$$

Then, using fractional powers and operators theory the authors get some existence result based on a priori bounded estimates for compact operators.

Other authors have studied problems involving impulsive act, for retarded and neutral functional differential equations we cite [7-17], for applications of impulsive differential equations on biology and neural networks we cite [18-21]. On the other hand impulsive fractional differential equations is a topic treated in [22,23].

In this article, the study of a class of neutral impulsive integro-differential equations is proposed. To get our results, we used the technique involving the fixed point theory of compact and condensing operators. We pointed out that the problem studied in this article has not been considered in the literature, once that the approach used in this study is totally different from those studies mentioned above. Actually, the main 
difference is that in our study we need to use an assumptions of compactness on the nonlinear equation, and in applications, these assumptions make all differences, because, even in infinite dimensional Hilbert space it is not straightforward handedly with compact sets. However, in our applications, we overcome this difficult using a well-known criterion of compactness in $L^{p}(\Omega)$ space [[24], Kolmogorov-Riesz-Weil theorem]. This is the principal motivation of this study.

We now turn to a summary of this study. The second section provides tools which are necessary to establish the main results that are the Theorems 2.3 and 2.4. In third section, we apply our abstract results in concrete examples.

\section{Preliminaries}

In this study, the symbols $\left(\mathcal{K},\|\cdot\|_{\mathbb{K}}\right)$ and $\left(\mathbb{Y},\|\cdot\|_{\curlyvee}\right)$ stand for Banach spaces with their, respectively, norms and we denote by $\mathcal{L}(\mathbb{Y}, \mathcal{X})$ the Banach space of bounded linear operators from $\mathbb{Y}$ into $\mathcal{X}$ endowed with the uniform operator topology; particularly, we denote $\mathcal{L}(\mathbb{X})$ when $\mathbb{Y}=\mathfrak{X}$. we start defining the evolution operator associated with the family $A(t), t \in[0 . b]$.

Definition 2.1. A family of operators $U(t, s), t \geq s, t, s \in I$ is said to be an evolution family associated to the problem (2.1) if the following conditions hold:

(a) $U(t, s) U(s, r)=U(t, r)$ for all $r \leq s \leq t$.

(b) For each $x \in \mathbb{X}$, the function $(t, s) \rightarrow U(t, s) x$ is continuous from $\{(t, s), t \geq s, t$, $s \in I\}$ into $\mathbb{X}$.

(c) For each $t>s$, the function $t \rightarrow U(t, s)$ is continuous differentiable with respect to $t$ and $\frac{\partial}{\partial t} U(t, s)=A(t) U(t, s)$.

The family of evolution system $U(t, s)$ is called exponential stable if there are positive constants $\tilde{M}$ and $\alpha$ such that $\|U(t, s)\|_{\mathcal{L}(\mathcal{X})} \leq \widetilde{M} e^{-\alpha(t-s)}$, for every $t, s \in[0, b]$.

Throughout this study, $A(t): D(A(t)) \subset \mathbb{X} \rightarrow \mathbb{X}$ denotes a family of unbounded closed linear operators defined in a common domain $D$, which is independent of $t$ and dense in $\mathfrak{X}$. Moreover, we assume that the system

$$
\left\{\begin{array}{l}
u^{\prime}(t)=A(t) u(t), t \geq s, t, s \in I \\
u(s)=x \in \mathbb{K}
\end{array}\right.
$$

has an associated evolution family of operators $U(t, s), t \geq s, t, s \in I$. For additional details and more properties about the family $U(t, s)$, we refer the reader to $[6,25,26]$.

To study the problem (2.1), we consider the space of normalized piecewise continuous functions $\mathcal{P C}([0, \tau], \mathbb{X})$, this means that, a function $u:[0, \tau] \rightarrow \mathbb{X}$ belongs to $\mathcal{P C}([0, \tau], \mathbb{X})$ if $u$ is continuous at $t \neq t_{i}, u\left(t_{i}^{-}\right)=u\left(t_{i}\right)$ and $u\left(t_{i}^{+}\right)<\infty$, for all $i=1, \ldots$, $m$. It is well known that if it is equipped with the norm $\|u\|_{\mathcal{P C}}=\sup _{s \in[0, a]}\|u(s)\|_{\text {久 }}$, then $\mathcal{P C}([0, \tau], \mathcal{X})$ became a Banach space.

The technique used in this study is based on the compactness criterion. For this reason, we will make the following assumptions.

Put $t_{0}=0, t_{n+1}=\tau$ and for $u \in \mathcal{P C}([0, \tau], \mathbb{X})$ we denote by $\tilde{u}_{i} \in P\left(\left[t_{i}, t_{i+1}\right] ; \mathbb{X}\right), i=0, \ldots, n$, the function given by 


$$
\tilde{u}_{i}(t)= \begin{cases}u(t), & \text { for } t \in\left(t_{i}, t_{i+1}\right] \\ u\left(t_{i}^{+}\right), & \text {for } t=t_{i}\end{cases}
$$

In particular, $\widetilde{B}_{i}$ stands the set defined by $\widetilde{B}_{i}=\left\{\widetilde{u}_{i}, u \in B\right\}$, where $B \subset \mathcal{P C}([0, \tau], \mathbb{X})$.

Lemma 2.1. ([12]) A set $B \subset \mathcal{P C}$ is relatively compact in $\mathcal{P C}$ if and only if the set $\tilde{B}_{i}$ is relatively compact in the space $C\left(\left[t_{i}, t_{i+1}\right] ; \mathbb{K}\right)$, for every $i=0,1, \ldots, n$.

The next step is to define the phase space. This will be done in the following way. The space $\mathcal{B}$, will be formed by all measurable functions $\varphi:(-\infty, 0] \rightarrow \mathcal{X}$ with seminorm $\|\cdot\|_{\mathcal{B}}$. On the phase space $\mathcal{B}$ we assume the following condition. Let $x:(-\infty, b] \rightarrow \mathbb{X}, b>0$ be a function such that $x_{0}=\phi, \varphi \in \mathcal{B}$ and $\left.x_{[0, b]} \in \mathcal{P C}[0, b] ; \mathcal{X}\right)$. Then the following properties hold true.

(i) $x_{t}$ is in $\mathcal{B}$;

(ii) $\|x(t)\|_{\mathcal{X}} \leq H\left\|x_{t}\right\|_{\mathcal{B}}$;

(iii) $\left\|x_{t}\right\|_{\mathcal{B}} \leq K(t-\sigma) \sup \left\{\|x(s)\|_{\mathfrak{X}}: \sigma \leq s \leq t\right\}+M(t-\sigma)\left\|x_{\sigma}\right\|_{\mathcal{B}}$, where $H>0$ is a constant; $K, M:[0, \infty) \rightarrow[1, \infty), K(\cdot)$ is continuous, $M(\cdot)$ is locally bounded and $H$, $K, M$ are independent of $x(\cdot)$.

Remark 2.1. To treat retarded impulsive differential equation we suitable modified the axioms of the abstract phase space $\mathcal{B}$. Actually, we drop the condition of continuity of the $\mathcal{B}$-valued function $t \rightarrow x_{t}$, since $t \rightarrow x(t) \in \mathcal{P C}$ in not a continuous function.

Following the ideas of [15], we used the notations $\bar{K}$ and $\bar{M}$ which is defined by

$$
\bar{K}:=\sup _{s \in[0, b]} K(s) \text { and } \bar{M}:=\sup _{s \in[0, b]} M(s) .
$$

In what following we give some examples of phase spaces whose the above axioms are satisfied.

Example 2.1. Consider the function $g(\theta)=e^{\gamma \theta}, \theta \leq 0, \gamma \geq 0$, and let $L^{2}([0, \pi], \mathbb{R})$ be the space of square integrable Lebesgue measure functions endowed with the norm $\|\xi\|_{L^{2}}=\left(\int_{0}^{\pi}|\xi(x)|^{2} d x\right)^{1 / 2}$. Then we define the phase space norm $\mathcal{B}$ as being

$$
\left.\mathcal{B}=\left\{\phi:(-\infty, 0] \rightarrow L^{2}([0, \pi] ; \mathbb{R}) ; \sup _{\theta \leq 0} e^{\gamma \theta}\|\phi(\theta)\|_{L^{2}}<\infty\right)\right\} .
$$

If $\mathcal{B}$ is endowed with the norm $\|\phi\|_{\mathcal{B}}=\sup _{\theta \geq 0} e^{\gamma \theta}\|\phi(\theta)\|_{L^{2}}$, for all $\phi \in \mathcal{B}$ then it is well known that $\left(\mathcal{B},\|\cdot\|_{\mathcal{B}}\right)$ is a phase space and the conditions $(i)$-(iii) are fulfilled. In these particular example it is possible to show that $H=1, K(t)=1$ and $M(t)=e^{-\gamma t}$, for all $t \geq 0$.

Motivated by Pazy [16,25] we adopt the following concept of mild solution to problem (1.1)-(1.3).

Definition 2.2. A function $u:(-\infty, b] \rightarrow \mathbb{X}, b>0$, is a local mild solution of problem (1.1)-(1.3) if the following conditions holds.

(i) $u_{0}=\varphi, \phi \in \mathcal{B}$; 
(ii) the function $u(t) \in \mathcal{P C}([0, b] ; \mathcal{X}), x\left(t_{i}^{+}\right)=x\left(t_{i}\right)+I_{i}\left(x_{t_{i}}\right)$, for all $i=1, \ldots, m$;

(iii) the integral equation below is satisfied,

$$
\begin{aligned}
u(t)= & U(t, 0)(\phi(0)+g(0, \phi))-g\left(t, u_{t}\right) \\
& +\int_{0}^{t} U(t, s) f\left(s, u_{s}, \int_{0}^{s} e\left(s, \tau, u_{\tau}\right) d \tau\right) \mathrm{d} s+\sum_{t_{i}<t} U\left(t, t_{i}\right) I_{i}\left(u_{t_{i}}\right), t \in[0, b],
\end{aligned}
$$

is satisfied.

The tools used in this study are based on point fixed theory. For this reason, the next two theorems play important role in the development of our results.

Theorem 2.1. [[27], Leray-Schauder Alternative] Let $C$ be a convex subset of a Banach space $\mathbb{X}$, and assume $0 \in C$. Let $F: C \rightarrow C$ be a completely continuous operator, and let

$$
\mathcal{E}(F):=\{x \in C ; x=\lambda F(x), \lambda \in(0,1)\} .
$$

Then either $\mathcal{E}(F)$ is unbounded or $F$ has a fixed point.

Theorem 2.2. [[28], Corollary 4.3.2] Suppose that D is a closed bounded convex subset of the Banach space $\mathbb{X}$ and that $B$ and $C$ are continuous function from $D$ to $\backslash$ with

(ma1) $B x+C x \in D$, for all $x \in D$.

(ma2) $\overline{C(D)}$ is compact set; and

(ma3) there is a number $0 \leq \gamma<1$ such that $\|B x-B y\| \leq \gamma\|x-y\|$, for all $x, y$ $\in D$.

Then there is $z \in D$ such that $B z+C z=z$.

Next, we stated some important conditions used in the proof of our results.

$\left(H_{1}\right)$ The function $g:[0, b] \times \mathcal{B} \rightarrow \mathbb{X}$ satisfy the following condition $\left(H_{1.1}\right)$ Let $\phi \in \mathcal{B}$ and consider the extension $y:(-\infty, b] \rightarrow \mathbb{X}$ of $\varphi$ which is given by

$$
\gamma(t)= \begin{cases}\phi(t), & t \leq 0, \\ U(t, 0) \phi(0), & t \in[0, b] .\end{cases}
$$

Then, each bounded set $B$ of $\mathcal{P C}$ the family of functions $\left\{g\left(t, y_{t}+u_{t}\right), t \in[0, b], u \in\right.$ $B\}$ is equi-continuous.

$\left(H_{1.2}\right)$ There are constants $c_{1}$ and $c_{2}$ such that $\|g(t, \phi)\| \leq c_{1}\|\phi\|_{\mathcal{B}}+c_{2}$, for all $t \geq 0$ and $\phi \in \mathcal{B}$.

$\left(H_{2}\right)$ The function $f:[0, b] \times \mathcal{B} \times \mathbb{X} \rightarrow \mathbb{X}$ satisfies the following conditions.

$\left(H_{2.1}\right)$ The function $(x, \varphi) \rightarrow f(t, \varphi, x)$ is continuous for almost everywhere $t \in$ $[0, b]$.

$\left(H_{2.2}\right)$ The function $t \rightarrow f(t, \varphi, x)$ is strong measurable for each $(\phi, x) \in \mathcal{B} \times \mathbb{X}$.. 
$\left(H_{2.3}\right)$ There is a positive continuous function $m:[0, b] \rightarrow[0, \infty)$ and a nondecreasing positive continuous function $\psi: \mathbb{R} \rightarrow[0, \infty)$ such that

$\|f(t, \phi, x)\|_{\mathfrak{X}} \leq m(t) \psi\left(\|\phi\|_{\mathcal{B}}+\|x\|_{\mathfrak{X}}\right)$,

for every $(t, \phi, x) \in[0, b] \times \mathcal{B} \times \mathbb{X}$.

$\left(H_{3}\right)$ The function $e:[0, b] \times[0, b] \times \mathcal{B} \rightarrow \mathbb{X}$ satisfy the following conditions.

$\left(H_{3.1}\right)$ The function $\varphi \rightarrow e(t, s, \varphi)$ is continuous almost everywhere for all $t, s \in$ $[0, b]$.

$\left(H_{3.2}\right)$ The function $(t, s) \rightarrow e(t, s, \varphi)$ is strong measurable for each $\phi \in \mathcal{B}$.

$\left(H_{3.3}\right)$ There is a positive continuous function $p:[0, b] \rightarrow[0, \infty)$ and a nondecreasing integrable positive function $\Omega: \mathbb{R} \rightarrow[0, \infty)$ such that

$\|e(t, s, \phi)\|_{\mathcal{X}} \leq p(s) \Omega\left(\|\phi\|_{\mathcal{B}}\right)$,

for all $(t, s, \phi) \in[0, b] \times[0, b] \times \mathcal{B}$.

$\left(H_{4}\right) \quad$ For each function $u:(-\infty, b] \rightarrow \mathcal{X}$, with $u_{0} \in \mathcal{B} \quad$ and $u(\cdot)_{\mid[0, b]} \in \mathcal{P C}([0, b] ; \mathbb{X}), \tau \rightarrow e\left(s, \tau, u_{\tau}\right), \tau \in[0, b]$ and $t \rightarrow f\left(t, u_{t}, x\right), t \in[0, b]$ are measurable functions for almost everywhere $s \in[0, b]$ and $x \in \mathbb{X}$.

Now we are already to state and prove the main result of this article.

Theorem 2.3. Assume that the conditions $\left(H_{1}\right)-\left(H_{4}\right)$ are satisfied. In addition, suppose that the following assumptions hold.

(t1) The function $g:[0, b] \times \mathcal{B} \rightarrow \mathbb{X}$ is completely continuous.

(t2) The operators $I_{i}$ are completely continuous and there are positive constants, $L_{i}$ such that

$$
\left\|I_{i}(x)\right\|_{\mathcal{X}} \leq L_{i}\|\phi\|_{\mathcal{B}}
$$

for all $\phi \in \mathcal{B}, x \in \mathcal{X}$, and $i=1, \ldots, m$.

(t3) For each bounded subsets $B \subset \mathcal{P C}([0, b]$; X) and $G \subset \mathbb{X}$ the set

$$
\left\{U(t, s) f\left(s, \bar{x}_{s}+y_{s}, z\right), x \in B, z \in G\right\},
$$

is relatively compact for each $t \geq s, t, s \in[0, b]$, where $\bar{x}:(-\infty, b] \rightarrow$ 久 is an extension of $x$ in such manner that $\bar{x}(t)=0, t \leq 0$ and $\bar{x}(t)=x(t), t \in[0, b]$.

$$
\begin{aligned}
& \text { If } 1-\bar{K}\left(\tilde{M} \sum_{i=1}^{m} L_{i}\right)>0 \text { and } \\
& \qquad \int_{0}^{t} \xi(s) \mathrm{d} s<\int_{\tilde{C}}^{\infty} \frac{\mathrm{d} s}{\psi(s)+\Omega(s)} \mathrm{d} s,
\end{aligned}
$$

where

$$
\widetilde{C}=\frac{(\bar{K} \widetilde{M}+\bar{K} \widetilde{M}+\bar{M})\|\phi\|+c_{2}(\widetilde{M}+1)}{1-\bar{K}\left(\widetilde{M} \sum_{i=1}^{m} L_{i}\right)}
$$


and

$$
\xi(t)=\max \left\{\frac{(\bar{K} \widetilde{M}+\bar{K} \widetilde{M}+M)\|\phi\|}{1-\widetilde{K}\left(\widetilde{M} \sum_{i=1}^{m} L_{i}\right)} \widetilde{K} \widetilde{M} m(t), p(t)\right\}, t \in[0, b],
$$

then, the problems (1.1)-(1.3) have a mild solution.

Proof. Suppose that $u:(-\infty, b] \rightarrow \mathbb{X}$ is a solution of (1.1)-(1.3) and let $y:(-\infty, b] \rightarrow \mathbb{X}$ be a continuous extension of $\varphi$ given in $\left(H_{1}\right)$. If we written the solution $u(\cdot)$ of the problem (1.1)-(1.3) as $u(t)=x(t)+y(t), t \in(-\infty, b]$, then we can see that $x(t)=0, t \leq 0$ and for $t \in[0, b]$ the following integral equation hold true

$$
\begin{aligned}
x(t)= & U(t, 0) g(0, \phi)-g\left(t, x_{t}+y_{t}\right) \\
& +\int_{0}^{t} U(t, s) f\left(s, x_{s}+y_{s}, \int_{0}^{s} e\left(s, \tau, x_{\tau}+y_{\tau}\right) d \tau\right) \mathrm{d} s \\
& +\sum_{t_{i}<t} U\left(t, t_{i}\right) I_{i}\left(x_{t_{i}}+y_{t_{i}}\right), \quad t \in[0, b] .
\end{aligned}
$$

Motivated by this remark we consider the space

$$
\Lambda=\left\{x:(-\infty, b] \rightarrow \mathbb{K} ; x(\theta)=0, \theta \leq 0, \text { and } x(\cdot)_{\mid[0, b]} \in \mathcal{P C}([0, b] ; \mathfrak{X})\right\},
$$

endowed with the norm $\|x\|_{\Lambda}=\sup _{t \in[0, b]}\|x(t)\|_{\text {久. }}$. Moreover, on $\Lambda$ we define the operators $\Gamma_{i}: \Lambda \rightarrow \Lambda, i=1,2,3$ given by

$$
\begin{aligned}
& \Gamma_{1} x(t)=U(t, 0) f(0, \phi)-f\left(t, x_{t}+y_{t}\right) \\
& \Gamma_{2} x(t)=\int_{0}^{t} U(t, s) g\left(s, x_{s}+y_{s}, \int_{0}^{s} e\left(s, \tau, x_{\tau}+y_{\tau}\right) d \tau\right) \mathrm{d} s \\
& \Gamma_{3} x(t)=\sum_{t_{i}<t} U\left(t, t_{i}\right) I_{i}\left(x_{t_{i}}+y_{t_{i}}\right),
\end{aligned}
$$

for all $t \in[0, b]$. Using the fact that $(U(t, s))_{t \geq s}$ is a evolution family of operators and assuming the conditions on $f, g$ and the family of operator $I_{i}, i=1, \ldots, m$, it is not difficult see that $t \rightarrow \Gamma_{i}(t), t \in[0, b]$ is a normalized piecewise continuous function for all $i=1, \ldots, m$. This shows that $\Gamma$ is well defined. In the next, we prove that the operator $\Gamma=\Gamma_{1}+\Gamma_{2}+\Gamma_{3}$ satisfies all conditions of Theorem 2.1. As the proof is very long we split it into various steps.

\section{Step 1. The operator 1 is completely continuous}

Let $x_{n} \in \Lambda, n \in \mathbb{N}$ be a sequence of elements of $\Lambda$ such that $x_{n} \rightarrow x$ as $n \rightarrow \infty$ for some $x \in \Lambda$. From the boundedness of operators $U(t, s)$ and the axioms of the phase space $\mathcal{B}$ it is easy to see that the set $\left\{x_{t}^{n}, x_{t}, n \in \mathbb{N}, t \in[0, b]\right\}$ is bounded in $\mathcal{B}$, which implies from condition $\left(H_{1}\right)$ the uniformity convergence of

$$
g\left(t, x_{t}^{n}+y_{t}\right) \rightarrow g\left(t, x_{t}+y_{t}\right), \text { as } n \rightarrow \infty
$$

on $[0, b]$. Thus, we have the continuity of $\Gamma_{1}$. From condition $(H 1)$, and the axiom (iii) follows immediately the $\Gamma_{1}$ applies bounded sets of $\Lambda$ into equi-continuous sets of $\Lambda$. On the other hand, again by (iii), and using the fact that $f$ is a completely 
continuous function, soon as infers that, for each $t \in[0, b]$, the set $\left\{g\left(t, x_{t}+y_{t}\right), x \in B\right\}$ is compact in $\mathfrak{X}$. The proof that $\Gamma_{1}$ is a completely continuous operator is complete.

\section{Step 2. The operator $\Gamma_{2}$ is complete continuous}

The condition $\left(H_{3.1}\right)$ permit us conclude that $e\left(t, s, x_{n}^{s}\right) \rightarrow e\left(t, s, x_{s}\right)$ as $n \rightarrow \infty$ almost everywhere for $t, s \in[0, b]$. By $\left(H_{3.3}\right)$, and the Lebesgue's dominated convergence theorem we conclude that

$$
\int_{0}^{t} e\left(t, s, x_{n}^{s}\right) \mathrm{d} s \rightarrow \int_{0}^{t} e\left(t, s, x_{s}\right) \mathrm{d} s
$$

uniformly for $t \in[0, b]$. From the strong continuity of the operators $(U(t, s))_{t \geq s}$, we can conclude that

$$
\int_{0}^{t} U(t, s) f\left(s, x_{s}^{n}+y_{s}, \int_{0}^{s} e\left(s, \tau, x_{\tau}^{n}+y_{\tau}\right) d \tau\right) \mathrm{d} s \rightarrow \int_{0}^{t} U(t, s) f\left(s, x_{s}+y_{s}, \int_{0}^{s} e\left(s, \tau, x_{\tau}+y_{\tau}\right) d \tau\right) \mathrm{d} s,
$$

as $n \rightarrow \infty$, uniformly for $t \in[0, b]$. This fact and the properties of the evolution family $U(t, s)$ lead us to the continuity of the operator $\Gamma_{2}$. Next, we show that $\Gamma_{2}$ takes bounded sets into equi-continuous sets. First, we observe from conditions $\left(H_{2.3}\right),\left(H_{3.3}\right)$ and the axioms of phase space that

$$
\left\{e\left(t, s, x_{s}+y_{s}\right), t, s \in[0, b], x \in B\right\},
$$

and

$$
\left\{f\left(t, x_{t}+y_{t}, \int_{0}^{t} e\left(t, s, x_{s}+y_{s}\right) \mathrm{d} s\right) ; t, s \in[0, b], x \in B\right\}
$$

are bounded sets in $\mathbf{X}$.

Let $\varepsilon>0$ be the arbitrary positive real number and $t_{1}, t_{2} \in[0, b], t_{1}>t_{2}$. Thus, take into account the previous notes and using the assumption (iii) we see that the set

$$
\left\{U\left(t_{2}-\varepsilon, s\right) g\left(s, x_{s}+y_{s}, \int_{0}^{s} e\left(s, \tau, x_{\tau}+y_{\tau}\right) d \tau\right) \mathrm{d} s, s \in\left[0, t_{2}-\varepsilon / 2\right], x \in B\right\}
$$

is relatively compact in $\mathfrak{X}$. Thus we have

$$
\begin{array}{r}
\leq \int_{0}^{t_{2}-\varepsilon / 2}\left\|\left[U\left(t_{1}, t_{2}-\varepsilon\right)-U\left(t_{2}, t_{2}-\varepsilon\right)\right] U\left(t_{2}-\varepsilon, s\right) g\left(s, x_{s}+y_{s}, \int_{0}^{s} e\left(s, \tau, x_{\tau}+y_{\tau}\right) d \tau\right)\right\| \mathrm{d} s \\
+\int_{t_{2}}^{t_{2}-\varepsilon / 2}\left\|\left[U\left(t_{1}, s\right)-U\left(t_{2}, s\right)\right] g\left(s, x_{s}+\gamma_{s} \int_{0}^{s} e\left(s, \tau, x_{\tau}+y_{\tau}\right) d \tau\right)\right\| \mathrm{d} s \\
+\int_{t_{2}}^{t_{1}}\left\|U\left(t_{1}, s\right) g\left(s, x_{s}+\gamma_{s}, \int_{0}^{s} e\left(s, \tau, x_{\tau}+y_{\tau}\right) d \tau\right)\right\| \mathrm{d} s,
\end{array}
$$


thus, from the continuity of $U(t, s)$ and the assumptions of compactness contained on the condition ( $t 3)$ we can infer the existence of $0<\delta<\varepsilon$ such that if $\left|t_{1}-t_{2}\right|<\delta$ then

$$
\begin{aligned}
\left\|\Gamma_{2} x\left(t_{1}\right)-\Gamma_{2} x\left(t_{2}\right)\right\| & \leq \varepsilon\left(t_{2}-\varepsilon / 2\right) \\
& +2 \varepsilon / 2 \widetilde{M} \sup _{s \in[0, b]}\left\|f\left(s, x_{s}+y_{s}, \int_{0}^{s} e\left(s, \tau, x_{\tau}+y_{\tau}\right) d \tau\right)\right\| \mathrm{d} s \\
& +\varepsilon \widetilde{M} \sup _{s \in[0, b]}\left\|f\left(s, x_{s}+y_{s}, \int_{0}^{s} e\left(s, \tau, x_{\tau}+y_{\tau}\right) d \tau\right)\right\| \mathrm{d} s .
\end{aligned}
$$

This shows the equi-continuity of $\Gamma_{2}$. In what follows, we show that for each $t \in[0$, b] the set

$$
\Theta(t)=\left\{\int_{0}^{t} U(t, s) f\left(s, x_{s}+y_{s}, \int_{0}^{s} e\left(s, \tau, x_{\tau}+y_{\tau}\right) d \tau\right) \mathrm{d} s, x \in B\right\},
$$

where $B \in \Lambda$, is pre-compact in $\Lambda$. To do that, we observe from (2.3) that for each $s$ $\in[0, t]$ the set

$$
\left\{f\left(s, x_{s}+y_{s}, \int_{0}^{s} e\left(s, \tau, x_{\tau}+y_{\tau}\right) d \tau\right), x \in B\right\}
$$

is a bounded set. Then,

$$
\begin{aligned}
\int_{0}^{t} U(t, s) f\left(s, x_{s}+y_{s} \int_{0}^{s} e\left(s, \tau, x_{\tau}+y_{\tau}\right) d \tau\right) \mathrm{d} s & =\int_{0}^{t-\varepsilon} U(t, s) f\left(s, x_{s}+y_{s} \int_{0}^{s} e\left(s, \tau, x_{\tau}+y_{\tau}\right) d \tau\right) \mathrm{d} s \\
& +\int_{t-\varepsilon}^{t} U(t, s) f\left(s, x_{s}+y_{s}, \int_{0}^{s} e\left(s, \tau, x_{\tau}+y_{\tau}\right) d \tau\right) \mathrm{d} s,
\end{aligned}
$$

which implies by [[28], Lemma 1.3]

$$
\begin{aligned}
& \int_{0}^{t} U(t, s) f\left(s, x_{s}+y_{s,} \int_{0}^{s} e\left(s, \tau, x_{\tau}+y_{\tau}\right) d \tau\right) \mathrm{d} s \\
& \in t \widetilde{c o}\left\{U(t, s) f\left(s, x_{s}+y_{s}, \int_{0}^{s} e\left(s, \tau, x_{\tau}+y_{\tau}\right) d \tau\right), s \in[0, t]\right\}+C_{\varepsilon},
\end{aligned}
$$

with $\operatorname{diam}\left(C_{\varepsilon}\right)<\varepsilon$, where $\operatorname{diam}(\cdot)$ denotes the diameter of the set $C_{\varepsilon}$ and $c o\{\cdot\}$ the convex hull. Taking all this into account we see that for each fixed $t \in[0, b]$, the set $\Theta(t)$ in (2.4) is relatively compact set in $\mathbb{X}$. This completes the proof that the operator $\Gamma_{2}$ is completely continuous.

\section{Step 3. The operator $\Gamma_{3}$ is completely continuous}

To show that is $\Gamma_{3}$ is a completely continuous, consider a bounded subset $B$ of $\Lambda$ and for each $i=1, \ldots, m$, define the set $\widetilde{\Pi}_{i} \subset \mathcal{C}\left(\left[t_{i}, t_{i+1}\right] ; \mathfrak{X}\right)$ as 


$$
\widetilde{\Pi}_{i}=\left\{\sum_{j=1}^{i-1} U\left(t, t_{j}\right) I_{j}\left(x_{t_{j}}\right), t \in\left[t_{i}, t_{i+1}\right], x \in \widetilde{B}_{i}\right\} .
$$

To prove that the sets $\widetilde{\Pi}_{i}, i=1, \ldots, m$, are precompacts in $\mathcal{C}\left(\left[t_{i}, t_{i+1}\right] ; \mathbb{K}\right)$, consider $t_{1}$, $t_{2} \in\left(t_{i}, t_{i+1}\right], t_{1}>t_{2}$. Using the continuity of $(t, s) \rightarrow U(t, s) x$, and the compactness of sets $I_{j}(B), j=1, \ldots, m$, given $\varepsilon>0$ there is $0<\delta<\varepsilon$ such that if $\left|t_{1}-t_{2}\right|<\delta$ we have

$$
\left\|\sum_{j=1}^{i}\left(U\left(t_{1}, t_{j}\right)-U\left(t_{2}, t_{j}\right)\right) I_{j}\left(x_{t_{j}}\right)\right\| \leq n \varepsilon,
$$

uniformly for $x \in B$. On the other hand for $t \in\left(t_{i}, t_{i+1}\right)$ fixed, from our hypothesis it is not difficult see that the set

$$
\widetilde{\Pi}_{i}(t)=\left\{\sum_{j=1}^{i} U\left(t, t_{j}\right) I_{j}\left(x_{t_{j}}\right), x \in B\right\},
$$

is relatively compact in $\mathcal{X}$

On the other hand, if $t=t_{i}$, the set $\widetilde{\Pi}_{i}\left(t_{i}\right)$ became

$$
\widetilde{\Pi}_{i}\left(t_{i}\right)=\left\{\sum_{j=1}^{i-1} U\left(t, t_{j}\right) I_{j}\left(x_{t_{j}}\right), x \in B .\right\},
$$

and proceeding as in the early case we infer that the set $\widetilde{\Pi}_{i}\left(t_{i}\right)$ is relatively compact in $\mathbb{X}$, The prove that the set

$$
\sum_{j=1}^{i-1}\left(U\left(t_{i}, t_{j}\right)-U\left(t_{2}, t_{j}\right)\right) I_{j}\left(x_{t_{j}}\right)
$$

is an equi-continuous set of functions is done in the same manner as at the beginning

of the section. The proof that $\Gamma_{3}$ is completely continuous is finished.

In the next, we obtain a priori estimative of the solutions for the equation $\lambda \Gamma x_{\lambda}=x_{\lambda}$, for $\lambda \in(0,1)$ and $=\Gamma=\Gamma_{1}+\Gamma_{2}+\Gamma_{3}$. Let $x$ be a solution of the equation $\lambda \Gamma\left(x_{\lambda}\right)=x_{\lambda}$, in addition we use the notation $m_{\lambda}(t)=\bar{K} \sup _{s \in[0, t]}\|x(s)\|+(\bar{K} \widetilde{M}+\bar{M})\|\phi\|_{\mathcal{B}}$, then we have

$$
\begin{aligned}
& \|x(t)\| \leq\|U(t, 0)\|_{\mathcal{L}(\mathfrak{X})}\|g(0, \phi)\|+\left\|g\left(t, x_{t}+y_{t}\right)\right\| \\
& +\int_{0}^{t}\|U(t, s)\|_{\mathcal{L}(\mathfrak{X})}\left\|f\left(s, x_{s}+\gamma_{s}, \int_{0}^{s} e\left(s, \tau, x_{\tau}+y_{\tau}\right) d \tau\right) \mathrm{d} s\right\| \\
& +\sum_{t_{j}<t}\left\|U\left(t, t_{j}\right)\right\|_{\mathcal{L}(\mathfrak{X})}\left\|I_{j}\left(x_{t_{j}}\right)\right\|_{\mathcal{L}(\mathfrak{X})} \\
& \leq \tilde{M}\|\phi\|+c_{1} m_{\lambda}(t)+c_{2}(\tilde{M}+1) \\
& +\tilde{M} \int_{0}^{t} m(s) \psi\left(m_{\lambda}(s)+\int_{0}^{s} p(\tau) \Omega\left(m_{\lambda}(\tau)\right) d \tau\right) \mathrm{d} s+\tilde{M} \sum_{t_{i}<t} L_{i} m_{\lambda}(t),
\end{aligned}
$$


this implies that

$$
\begin{aligned}
m_{\lambda}(t) \leq & \frac{(\bar{K} \tilde{M}+\bar{K} \widetilde{M}+\bar{M})\|\phi\|+c_{2}(\tilde{M}+1)}{1-\bar{K}\left(\widetilde{M} \sum_{i=1}^{m} L_{i}\right)} \\
& +\frac{(\bar{K} \widetilde{M}+\bar{K} \widetilde{M}+\bar{M})\|\phi\|}{1-\bar{K}\left(\widetilde{M} \sum_{i=1}^{m} L_{i}\right)} \widetilde{M} \bar{K} \int_{0}^{t} m(s) \psi\left(m_{\lambda}(s)+\int_{0}^{s} p(\tau) \Omega\left(m_{\lambda}(\tau)\right) d \tau\right) \mathrm{d} s .
\end{aligned}
$$

If we take the right-hand side of the previous inequalities and call it of $v(t)$ we have that $m_{\lambda}(t) \leq v(t)$, for all $t \in[0, b]$. This leads us to the following inequality:

$$
\begin{aligned}
v(t) \leq & \frac{(\bar{K} \tilde{M}+\bar{K} \widetilde{M}+\bar{M})\|\phi\|+c_{2}(\widetilde{M}+1)}{1-\bar{K}\left(\widetilde{M} \sum_{i=1}^{m} L_{i}\right)} \\
& +\frac{(\bar{K} \widetilde{M}+\bar{K} \widetilde{M}+\bar{M})\|\phi\|}{1-\bar{K}\left(\widetilde{M} \sum_{i=1}^{m} L_{i}\right)} \widetilde{K} \int_{0}^{t} m(s) \psi\left(v(s)+\int_{0}^{s} p(\tau) \Omega(v(\tau)) d \tau\right) \mathrm{d} s,
\end{aligned}
$$

this yields

$$
v^{\prime}(t) \leq \frac{(\bar{K} \widetilde{M}+\bar{K} \widetilde{M}+\bar{M})\|\phi\|}{1-\bar{K}\left(\widetilde{M} \sum_{i=1}^{m} L_{i}\right)} \widetilde{K} \widetilde{M} m(t) \psi\left(v(t)+\int_{0}^{t} p(\tau) \Omega(v(\tau)) d \tau\right) .
$$

Next, we considered the function $\varpi(t)=v(t)+\int_{0}^{t} p(s) \Omega(v(s)) \mathrm{d} s$, thus we have that $v$ $(0)=\varpi(0)$ and $v(t) \leq \varpi(t)$, for all $t \in[0, b]$, using this and the non-decreasingly properties of the function $\psi(\cdot)$, we get

$$
\varpi^{\prime}(t) \leq \frac{(\bar{K} \widetilde{M}+\bar{K} \widetilde{M}+\bar{M})\|\phi\|}{1-\bar{K}\left(\widetilde{M} \sum_{i=1}^{m} L_{i}\right)} \bar{K} \widetilde{M} m(t) \psi(\varpi(t))+p(t) \Omega(\varpi(t)), t \in[0, b] .
$$

Observe that if we define the function $\xi(t)=\max \left\{\frac{(\widetilde{K} \widetilde{M}+\widetilde{K} \widetilde{M}+M)\|\phi\|}{1-\widetilde{K}\left(\widetilde{M} \sum_{i=1}^{m} L_{i}\right)} \widetilde{K} m(t), p(t)\right\}, t \in[0, b]$ then we have

$$
\varpi^{\prime}(t) \leq \xi(t) \psi(\varpi(t))+\Omega(\varpi(t))
$$

which implies that

$$
\frac{\varpi^{\prime}(t)}{\psi(\varpi(t))+\Omega(\varpi(t))} \leq \xi(t)
$$

for all $t \in[0, b]$. Integrating the early inequality from 0 to $t$ we have

$$
\int_{\varpi(0)}^{\varpi(t)} \frac{\mathrm{d} s}{\psi(s)+\Omega(s)} \leq \int_{0}^{t} \xi(s) \mathrm{d} s<\int_{\varpi(0)}^{\infty} \frac{\mathrm{d} s}{\psi(s)+\Omega(s)} .
$$

The early inequalities enable us to conclude that the set $\left\{x_{\lambda}, x_{\lambda}=\Gamma x_{\lambda}, \lambda \in(0,1)\right\}$ is bounded. From Theorem 2.1 the problem (1.1)-(1.3) has a mild solution. The proof of theorem is completed.

In the next result, the following conditions are used. 
$\left(\mathbf{G}_{\mathbf{1}}\right)$ There is a positive constant $L_{f}$ such that

$$
\|g(t, \phi)-g(t, \psi)\|_{\mathcal{X}} \leq L_{g}\|\phi-\psi\|_{\mathcal{B}},
$$

for every $t \in[0, b]$ and $\phi, \psi \in \mathcal{B}$.

$\left(\mathbf{G}_{2}\right)$ There are positive constants $d_{i}, i=1, \ldots, m$, such that

$$
\left\|I_{i}(x)-I_{i}(y)\right\|_{\text {久 }} \leq d_{i}\|x-y\|_{\text {久 }}
$$

for every $x, y \in \mathbb{X}$.

Theorem 2.4. Assume that the condition (H2)-(H3) and (G1)-(G2) are satisfied. In addition, suppose that the assumption (iii) of Theorem (2.3) is satisfied. Then if

$$
L_{g} \bar{K}+\bar{K} \widetilde{M} \liminf _{\xi \rightarrow \infty} \frac{\psi\left(\xi+\Omega(\xi) \int_{0}^{b} p(\tau) d \tau\right)}{\xi} \int_{0}^{b} m(s) \mathrm{d} s+\widetilde{M} \bar{K} \sum_{i=1}^{n} d_{i}<1,
$$

and

$$
\left(L_{g}+\widetilde{M} \sum_{i=1}^{n} d_{i}\right)<1,
$$

then the problem (1.1)-(1.3) has a mild solution.

Proof. Let us consider the operator $\Gamma: \Lambda \rightarrow \Lambda$ defined as in Theorem 2.3. We claim that there is $r>0$ such that $\Gamma\left(B_{r}\right) \subset B_{r}$. Suppose by contradiction that this assumption is false. Then for each $r>0$ there are $t_{r} \in[0, b]$ and $u_{r}(\cdot) \in B_{r}$ such that $\left\|\Gamma\left(u_{r}\right)\left(t_{r}\right)\right\|_{\mathfrak{x}} \geq r$. This implies that

$$
\begin{aligned}
r \leq & \tilde{M}\left(H\|\phi\|_{\mathcal{B}}+\|g(0, \phi)\|_{\mathfrak{X}}\right)+\tilde{M} \int_{0}^{t_{r}}\left\|f\left(s, u_{s}^{r}+y_{s} \int_{0}^{s} e\left(s, \tau, u_{\tau}^{r}+y_{\tau}\right) d \tau\right)\right\| \mathrm{d} s \\
& +\sum_{t_{i}<t_{r}}\left\|U\left(t_{r}, t_{i}\right) I_{i}\left(u_{t_{i}}^{r}+y_{t_{i}}\right)\right\|+\left\|g\left(t_{t_{r}}, u_{t_{r}}^{r}+y_{t_{r}}\right)\right\|_{\mathfrak{X}} \\
\leq & \tilde{M}\left(H\|\phi\|_{\mathcal{B}}+\|g(0, \phi)\| \mathfrak{X}\right)+L_{g}(\bar{K} r+(\bar{K} \tilde{M} H+\bar{M})\|\phi\|)+\sup _{s \in[0, b]}\|g(s, 0)\| \\
& +\widetilde{M} \psi\left(\bar{K} r+(\bar{K} \tilde{M} H+\bar{M})\|\phi\|+\Omega(\bar{K} r+(\bar{K} \tilde{M} H+\bar{M})\|\phi\|) \int_{0}^{b} p(\tau) d \tau\right) \int_{0}^{b} m(s) \mathrm{d} s \\
& +\widetilde{M} \sum_{i=1}^{n}\left(d_{i}(\bar{K} r+(\bar{K} \tilde{M} H+\bar{M})\|\phi\|)+\left\|I_{i}(0)\right\|_{\mathfrak{X}}\right),
\end{aligned}
$$

take the lim inf in the previous inequality, we have

$$
\begin{aligned}
1 \leq & L_{g} \bar{K}+\bar{K} \widetilde{M} \liminf _{\xi \rightarrow \infty} \frac{\psi\left(\xi+\Omega(\xi) \int_{0}^{b} p(\tau) d \tau\right)}{\xi} \int_{0}^{b} m(s) \mathrm{d} s \\
& +\tilde{M} \bar{K} \sum_{i=1}^{n} d_{i}
\end{aligned}
$$

which is contradictory with our assumptions. So let $r>0$ be such a number and consider the restriction $\Gamma_{\left.\right|_{B_{r}}}$ of the operator $\Gamma$ on $B_{r}$, that is, $\Gamma_{\left.\right|_{B_{r}}}: B_{r} \rightarrow B_{r}$ Next, we split the operator $\Gamma$ in the following way $\Gamma=\Gamma_{1}+\Gamma_{2}$, where 


$$
\begin{aligned}
& \Gamma_{1}(u)=U(t, 0)(\phi(0)+g(0, \phi))-g\left(t, u_{t}+y_{t}\right)+\sum_{t_{i}<t} U\left(t, t_{i}\right) I_{i}\left(u_{t_{i}}+y_{t_{i}}\right), t \in[0, b] \text { and } \\
& \Gamma_{2}(u)=\int_{0}^{t} U(t, s) f\left(s, u_{s}+y_{s} \int_{0}^{s} e\left(s, \tau, u_{\tau}+y_{\tau}\right) d \tau\right) \mathrm{d} s, t \in[0, b] .
\end{aligned}
$$

As shown in the proof of Theorem 2.3, it is not difficult to see that $\Gamma_{2}$ is completely continuous and for $u_{1}, u_{2} \in \Lambda$ we have that

$$
\begin{aligned}
\left\|\Gamma_{1}\left(u_{1}\right)(t)-\Gamma_{1}\left(u_{2}\right)(t)\right\|_{\aleph} & \leq L_{f}\left\|u_{t}^{1}-u_{t}^{2}\right\|+\widetilde{M} \sum_{i=1}^{n} d_{i}\left\|u_{t_{i}}^{1}-u_{t_{i}}^{2}\right\| \\
& \leq\left(L_{f}+\widetilde{M} \sum_{i=1}^{n} d_{i}\right)\left\|u^{1}-u^{2}\right\|_{\Lambda}
\end{aligned}
$$

The previous inequality shows that $\Gamma_{1}$ is contractive. Now, by Theorem 2.2, we can conclude that the problem (1.1)-(1.3) has a mild solution.

\section{Applications}

The main aim of this section is to apply our abstract results in concrete examples. To this end, we handle with a very special kind of operators. To be more specific, on the Banach space $\mathcal{X}=L^{2}([0, \pi], \mathbb{R})$ we define the operator $A: D(A) \rightarrow \mathbb{X}$ given by $A x(\xi)=$ $x^{\prime \prime}(\xi), \xi \in[0, \pi]$ with domain

$$
D(A)=\left\{x \in \mathbb{X} ; x^{\prime \prime} \in \text { Xand } x(0)=x(\pi)=0\right\} .
$$

It is well known that in this case $A$ has a discrete spectrum which is given by $-n^{2}, n$ $\in \mathbb{N}$. Moreover, $\mathbb{X}$ has a completely orthonormal base formed by eigenfunctions of $A$ associated with the eigenvalues $-n^{2}$, which is given $x_{n}(\xi)=\sqrt{\frac{2}{\pi}} \sin (n \xi), n \in \mathbb{N}$. This implies that the following conditions are satisfied.

(i) For each $f \in \mathbb{X}, f(\xi)=\sum_{n=1}^{\infty}\left\langle f, x_{n}\right\rangle x_{n}(\xi)$,

(ii) For each $f \in D(A)$, we have $A f(\xi)=-\sum_{n=1}^{\infty} n^{2}\left\langle f, x_{n}\right\rangle x_{n}(\xi)$,

where $\langle\cdot, \cdot\rangle$ represents the inner product in $\mathfrak{X}$. Taking into account all these information, it is possible to prove that the operator $A$ is the infinitesimal generator of a compact semigroup of bounded linear operators $(T(t))_{t \geq 0}$, which is given by

$$
T(t) f(\xi)=\sum_{n=1}^{\infty} e^{-n^{2} t}\left\langle f, x_{n}\right\rangle x_{n}(\xi), t \geq 0, \xi \in[0, \pi] .
$$

To guarantee the existence of an evolution family associated with the problem

$$
\left\{\begin{array}{l}
u^{\prime}(t)=A(t) u(t), t \geq s, t, s \in[0, b] \\
u(s)=x \in \mathbb{K}
\end{array}\right.
$$

the following assumptions on the function $a_{0}:[0, b] \times[0, \pi] \rightarrow \mathbb{R}$ are made 
$\left(\mathfrak{a}_{1}\right)$ There are constants $c>0$ and $\alpha \in(0,1)$ such that

$$
\left|a_{0}(t, \xi)-a_{0}(s, \xi)\right| \leq|t-s|^{\alpha},
$$

for all $t, s \in[0, b]$ and almost everywhere $\xi \in[0, \pi]$.

$\left(\mathfrak{b}_{0}\right)$ there is a real number $c_{0}$ such that

$$
a_{0}(\tau, \xi) \leq c_{0},
$$

for all $\tau \in[0, \infty)$ and $\xi \in[0, \pi]$.

Letting $D(A(t))=D(A)$ for all $t \geq 0$ and $A(t) x(\xi)=a_{0}(t, \xi) x^{\prime \prime}(\xi), \xi \in[0, \pi]$, we have that the system (3.1) has an associated evolution family of operators $(U(t, s))_{t \geq s}$ which is given explicitly by the following formula:

$$
(U(t, s) f)(\xi)=\sum_{n=0}^{\infty} e^{-n^{2} \int_{s}^{t} a_{0}(\tau, \xi) d \tau}\left\langle f, x_{n}\right\rangle x_{n}(\xi), \xi \in[0, \pi], t \in[0, b]
$$

Using the properties of semigroup $(T(t))_{t \geq 0}$ it is straightforward to show that $U(t, s)$ satisfies the condition

$$
\|U(t, s)\|_{\mathcal{L}(\mathrm{X})} \leq e^{-c_{0}(t-s)}, t \geq s .
$$

Next, we consider the following partial differential equations

$$
\left\{\begin{array}{l}
\frac{\partial}{\partial t}\left(u(t, \xi)+\int_{0}^{\xi} \int_{0}^{t} k_{1}\left(s, \xi_{1}\right) u\left(s, \xi_{1}\right) \sin (\xi) \mathrm{d} s d \xi_{1}\right) \\
=a_{0}(t, \xi) \frac{\partial^{2}}{\partial \xi^{2}}\left(u(t, \xi)+\int_{0}^{\xi} \int_{0}^{t} k_{1}\left(s, \xi_{1}\right) u\left(s, \xi_{1}\right) \sin (\xi) \mathrm{d} s d \xi_{1}\right) \\
\quad+\int_{-\infty}^{t} k_{2}(-s) P_{2}(s, u(t, \xi)) \mathrm{d} s+\int_{0}^{t} \int_{-\infty}^{s} k_{3}(t-\delta) P_{3}(\delta-t, u(t, \xi) d \delta \mathrm{d} s), \\
u(t, 0)=u(t, \pi)=0, s \in[0, \pi], \\
u(s, \xi)=\phi(s, \xi), s \leq 0, \xi \in[0, \pi], \\
u\left(t_{i}^{+}, \xi\right)=u\left(t_{i}, \xi\right)+\int_{0}^{\xi} k_{4, i}(\xi) u\left(t_{i}, \xi_{1}\right) d \xi_{1}, \quad i=1, \ldots, m .
\end{array}\right.
$$

To model the problem (3.2) we choose as the phase space the set formed by all piecewise continuous functions $\varphi:(-\infty, 0] \rightarrow \mathbb{X}$ which $\sup _{s \leq 0} h(\theta)\|\phi(s)\|<\infty$, where $h(\theta)=e^{\beta \theta}, \theta \leq 0$, and we denote this space by $\mathcal{B}_{h}$ equipping it with the norm $\|\varphi\|_{\mathcal{B}_{h}}=\sup _{s \leq 0} h(s)\|\varphi(s)\|$. In order to show that the conditions $\left(H_{1}\right)-\left(H_{4}\right)$ are satisfied we needed to consider the following assumptions.

$\left(\mathfrak{p}_{1}\right)$ The function $P_{2}:(-\infty, 0] \times \mathbb{R} \rightarrow[0, \infty)$ satisfies the following conditions: $\left(\mathfrak{p}_{1.1}\right)$ for each $\eta \in \mathbb{R}, s \rightarrow P_{2}(s, \eta)$ is a measurable and bounded function, $\left(\mathfrak{p}_{1.2}\right)$ there is a positive constant $L_{P_{2}}$ such that

$$
\left|P_{2}\left(s, \eta_{1}\right)-P_{2}\left(s, \eta_{2}\right)\right| \leq L_{P_{2}}\left|\eta_{1}-\eta_{2}\right|,
$$

for all $s \leq 0$, and $\eta_{i} \in \mathbb{R}, i=1,2$. 
$\left(\mathfrak{p}_{2}\right)$ The functions $s \rightarrow \int_{0}^{\pi} k_{1}\left(s, \xi_{1}\right) d \xi_{1}$ and $\xi \rightarrow \int_{-b}^{0} k_{1}(s, \xi) \mathrm{d} s$ are bounded almost everywhere on $[-b, 0] \times[0, \pi], s \rightarrow \int_{0}^{\pi} k_{1}\left(s, \xi_{1}\right)^{2} e^{-\beta s} d \xi_{1}$ is integrable on the interval $[-b, 0]$.

$\left(\mathfrak{p}_{3}\right) k_{2}(\cdot) \in L((-\infty, \pi])$ and $s \rightarrow P_{2}(s, \eta)$ is measurable and bounded function for each $\eta \in \mathbb{R}$. In addition we assume the existence of positive constant $L_{P_{2}}$ such that the following inequality hold true

$$
\left|P_{2}\left(s, \eta_{2}\right)-P_{2}\left(s, \eta_{1}\right)\right| \leq L_{P_{2}}\left|\eta_{2}-\eta_{1}\right|,
$$

for almost everywhere $s \in(-\infty, 0]$ and $\eta_{i} \in[0, \pi], i=1,2$.

$\left(\mathfrak{p}_{4}\right)$ The function $P_{3}:[-\pi, \infty) \times \mathbb{R} \rightarrow \mathbb{R}$ satisfies the following conditions.

$\left(\mathfrak{p}_{4.1}\right)$ for each $\eta \in \mathbb{R}, s \rightarrow P_{3}(s, \eta), s \in[-\infty, b)$, is a measurable and bounded function,

$\left(\mathfrak{p}_{4.2}\right)$ there is a positive constant $L_{P_{3}}$ such that

$\left|P_{3}\left(t, \eta_{2}\right)-P_{3}\left(t, \eta_{1}\right)\right| \leq L_{P_{3}}\left|\eta_{2}-\eta_{1}\right|$,

for all $s \in[-\pi, \infty)$ and $\eta_{i} \in \mathbb{R}, i=1,2$.

$\left(\mathfrak{p}_{4.3}\right)$ The function $k_{3}(\cdot) \in L([-\pi, \infty))$.

To transform the problem (3.2) into the abstract system (1.1), we define the functions $g:[0, b] \times \mathcal{B}_{h} \rightarrow \mathbb{X}, \quad f:[0, b] \times \mathcal{B} \times \mathbb{X} \rightarrow \mathbb{X}, \quad e:[0, T] \times[0, T] \times \mathcal{B}_{h} \rightarrow \mathbb{X}$ and $I_{i}: \mathcal{B} \rightarrow \mathfrak{X}, i=1,2, \ldots, n$, respectively, given by,

$$
\begin{aligned}
g(t, \varphi)(\xi) & =\int_{0}^{\xi} \int_{-t}^{0} \varphi\left(s, \xi_{1}\right) \sin (\xi) \mathrm{d} s, t \in[0, \infty), \xi \in[0, \pi] \\
f(t, \varphi, x)(\xi) & =\int_{-\infty}^{0} k_{2}(-s) P_{2}(s, \varphi(0, \xi)) \mathrm{d} s+x(\xi), t \in[0, b], \xi \in[0, \pi] \\
e(t, s, \varphi)(\xi) & =\int_{-\infty}^{s} k_{3}(t-\tau) P_{3}(\tau, \varphi(0, \xi)) d \tau, t, s \in[0, T], \xi \in[0, \pi] \\
I_{i}(\phi)(\xi) & =\int_{0}^{\xi} k_{4, i}(\xi) \phi\left(0, \xi_{1}\right) d \xi_{1} .
\end{aligned}
$$

We shall show that the condition $\left(H_{1}\right)$ hold true. In fact, let $x:(-\infty, \pi] \rightarrow L^{1}(0, \pi)$ be a bounded function such that $x \mid[0, \pi] \in \mathcal{P C}\left([0, \pi] ; L^{1}(0, \pi)\right)$ we have 


$$
\begin{aligned}
\left|g\left(t+h, x_{t+h}\right)(\xi)-g\left(t, x_{t}\right)(\xi)\right| & \leq \int_{0}^{\xi} \int_{t}^{t+h}\left|x\left(s, \xi_{1}\right) \sin (\xi)\right| \mathrm{d} s d \xi \\
& \leq \int_{t}^{t+h}\left(\int_{0}^{\xi} x\left(s, \xi_{1}\right)^{2} d \xi_{1}\right)^{1 / 2}\left(\int_{0}^{\xi} k_{1}\left(s, \xi_{1}\right)^{2} d \xi_{1}\right)^{1 / 2}|\sin (\xi)| \mathrm{d} s \\
& \leq \sup _{s \in[-b, 0]}\left(\int_{0}^{\pi} x\left(s, \xi_{1}\right)^{2} d \xi_{1}\right)^{1 / 2} \int_{t}^{t+h}\left(\int_{0}^{\pi} k_{1}\left(s, \xi_{1}\right)^{2} d \xi_{1}\right)^{1 / 2}|\sin (\xi)| \mathrm{d} s .
\end{aligned}
$$

The previous inequalities jointly with the assumption $\left(\mathfrak{p}_{2}\right)$ show that the function $t$ $\rightarrow g\left(t, x_{t}\right)$ is uniformly continuous on bounded subsets of $\mathcal{P C}\left([0, b], L^{2}(0, \pi)\right)$ which implies that the condition $\left(H_{1.1}\right)$ hold true. To prove that the condition $\left(H_{1.2}\right)$ is satisfied, we observe that

$$
\begin{aligned}
|g(t, \phi)(\xi)| & \leq \int_{0}^{\xi} \int_{-t}^{0}\left|k_{1}\left(s, \xi_{1}\right) \| \phi\left(s, \xi_{1}\right)\right| \mathrm{d} s d \xi_{1} \\
& \leq \int_{-t}^{0}\left(\int_{0}^{\xi} k_{1}\left(s, \xi_{1}\right)^{2} e^{-\beta s} d \xi_{1}\right)^{1 / 2}\left(\int_{0}^{\xi} \phi\left(s, \xi_{1}\right)^{2} e^{\beta s} d \xi_{1}\right)^{1 / 2} \mathrm{~d} s \\
& \leq \sup _{s \leq 0}\left(\int_{0}^{\xi} \phi\left(s, \xi_{1}\right)^{2} e^{\beta s} d \xi_{1}\right)^{1 / 2} \int_{-t}^{0}\left(\int_{0}^{\pi} k_{1}\left(s, \xi_{1}\right)^{2} e^{-\beta, s} d \xi_{1}\right)^{1 / 2} \mathrm{~d} s,
\end{aligned}
$$

which implies the condition $\left(H_{1.2}\right)$.

The next step is a proof that the function $(x, \varphi) \rightarrow f(t, \varphi, x)$ is continuous. However, with the help of condition $\left(\mathfrak{p}_{2}\right)$ we have

$$
\begin{aligned}
\int_{0}^{\pi}\left|f\left(t, \phi_{2}, x_{2}\right)(\xi)-f\left(t, \phi_{1}, x_{1}\right)(\xi)\right|^{2} \leq & 4 L_{P_{2}} \int_{0}^{\pi}\left|\phi_{2}(0, \xi)-\phi_{1}(0, \xi)\right|^{2} d \xi+4 \int_{0}^{\pi}\left|x_{2}(\xi)-x_{1}(\xi)\right|^{2} d \xi \\
\leq & 4 L_{P_{2}} \sup _{s \leq 0} \int_{0}^{\pi} e^{\beta s}\left|\phi_{2}(s, \xi)-\phi_{1}(s, \xi)\right|^{2} d \xi \\
& +4 \int_{0}^{\pi}\left|x_{2}(\xi)-x_{1}(\xi)\right|^{2} d \xi
\end{aligned}
$$

for all $\phi_{i} \in \mathcal{B}, x_{i} \in \mathbb{X}, i=1,2$. Thus we have shown that the condition $\left(H_{2.1}\right)$ is fulfilled. In particular, as $P_{2}$ is continuous in the second variable we have that for each $(\phi, x) \in \mathcal{B} \times \mathbb{X}$ fixed the function $\xi \rightarrow \int_{-\infty}^{0} k_{2}(-s) P_{2}(s, \phi(0, \xi)) \mathrm{d} s$ is measurable. Thus from [[24], Theorem 1.2.1] we infer that $t \rightarrow f(t, \varphi, x)$ is measurable for each $(\phi, x) \in \mathcal{B} \times \mathbb{X}$. On the other hand, assuming that $s \rightarrow P_{2}(s, 0), s \in(-\infty, 0]$ is bounded function, we have that

$$
|f(t, \phi, x)(\xi)|^{2} \leq 4 L_{P_{2}}|\phi(0, \xi)|\left(\int_{-\infty}^{0} k_{2}(-s) \mathrm{d} s\right)^{2}+16\left(\int_{-\infty}^{0} k_{2}(-s) P_{2}(s, 0) \mathrm{d} s\right)^{2}+16|x(\xi)|^{2}
$$


which implies that

$$
\begin{aligned}
\|f(t, \phi, x)\|_{\mathcal{X}} & \leq 4\left\{L_{P_{2}}\left(\int_{-\infty}^{0} k_{2}(-s) \mathrm{d} s\right)^{2},\right. \\
& \left.4 \pi\left(\int_{-\infty}^{0} k_{2}(-s) P_{2}(s, 0) \mathrm{d} s\right)^{2}, 4\right\}^{1 / 2}\left(\|\phi\|_{\mathcal{B}}+\|x\|_{\boldsymbol{X}}\right) .
\end{aligned}
$$

Thus the condition $\left(H_{2.3}\right)$ is fulfilled.

On the other hand, the same idea applied to prove that the previous functions is of Caratheádory type can be used to show that function $e(\cdot,, \cdot)$ satisfies the same property. Here it is mentioned that the functions that appear in the condition $\left(H_{3.3}\right)$ are given by

$$
\begin{aligned}
\|e(t, s, \phi)\|_{\mathcal{X}} \leq & 4 \max \left\{L_{P_{2}}\left(\int_{-\infty}^{s} k_{2}(-\delta) d \delta\right)^{2},\right. \\
& \left.4 \pi\left(\int_{-\infty}^{s} k_{2}(-\delta) P_{2}(\delta, 0) d \delta\right)^{2}, 4\right\}^{1 / 2}\|\phi\|_{\mathcal{B} .}
\end{aligned}
$$

Finally, it remains that the condition $(H 4)$ is valid. However, we observe that

$$
\mathcal{B}_{h} \subset L_{h}^{\infty}\left((-\infty, 0], L^{2}((0, \pi))\right),
$$

where a function $\varphi:(-\infty, 0] \rightarrow L^{2}(0, \pi)$ is an element of $L_{h}^{\infty}\left((-\infty, 0], L^{2}(0, \pi)\right)$ if and only if

$$
\text { ess } \sup _{s \leq 0} \int_{0}^{\pi} h(s) \phi^{2}(s, \xi) d \xi<\infty,
$$

with the norm defined by $\|\phi\|_{L_{h}^{\infty}}=\inf \left\{a ; \mu\left\{\theta \in(-\infty, 0] ;\|\phi\|_{L^{2}}>a\right\}=0\right\}$, where $\mu$ $=h d \xi$, and $d \xi$ representing the Lebesgue measure on $(-\infty, 0]$. Thus, following the ideas of [[29], Theorem 3.8] and using the fact that $h(\theta-t) \leq G(-t) h(\theta), \theta \leq 0, G(-t)=e^{-\beta t}, t$ $\geq 0$, we see that if $u:(0,-\infty] \rightarrow L^{2}(0, \pi)$ is admissible function in the sense of [29], then we derive the mensurability of $t \rightarrow u_{t}, t \in[0, b]$. Thus, as $e(\cdot,,$,$) and f(\cdot,,$,$) are$ measurable functions we infer that $\tau \rightarrow e\left(t, \tau, u_{\tau}\right)$ and $\tau \rightarrow f\left(t, u_{\tau}, x\right)$ for all $t, \tau \in[0$, $b], x \in L^{2}(0, \pi)$. Now we will see that the conditions of the Theorem 2.3 hold. To see this, we observe that

$$
\begin{aligned}
|g(t, \varphi)(\xi+h)-g(t, \varphi)(\xi)| & \leq \int_{0}^{\xi} \int_{-t}^{0}\left|\varphi\left(s, \xi_{1}\right) \|(\sin (\xi+h)-\sin (\xi))\right| \mathrm{d} s d \xi_{1} \\
& +\int_{\xi}^{\xi+h} \int_{-t}^{0}\left|\varphi\left(s, \xi_{1}\right) \| \sin (\xi)\right| \mathrm{d} s d \xi_{1},
\end{aligned}
$$


taking the advantage of the previous inequality we have

$$
\begin{aligned}
|g(t, \varphi)(\xi+h)-g(t, \varphi)(\xi)| \leq & b \sup _{s \leq 0}\left(\int_{0}^{\pi} e^{\beta s}\left|\varphi\left(s, \xi_{1}\right)\right|^{2} d \xi\right) \int_{0}^{\pi-h}|(\sin (\xi+h)-\sin (\xi))| \mathrm{d} s \\
& +b h \sup _{s \leq 0}\left(\int_{0}^{\pi} e^{\beta s}\left|\varphi\left(s, \xi_{1}\right)\right|^{2} d \xi\right)^{1 / 2}|\sin (\xi+h)| \mathrm{d} s
\end{aligned}
$$

which implies from [[24], Theorem A.5.2] the assumption (t1).

The same idea which was used to prove the compactness of the function $g$ can be used to prove that compactness of the operators $I_{i}, i=1, \ldots, m$. Regarding the inequality that appear in the condition ( $t 2)$, we observe that to exhibit explicitly the $c_{i}$ constants, $i$ $=1, \ldots, m$, the following account is necessary

$$
\left\|I_{i}(\phi)\right\|_{\mathcal{B}}^{2} \leq \pi\|\phi\|_{\mathcal{B}}^{2} \int_{0}^{\pi} k_{4, i}^{2}(\xi) d \xi,
$$

for all $i=1, \ldots, m$.

As we choose the phase space as being $\mathcal{B}_{h}$, then it is possible to show that the constant $H$ and the functions $K(\cdot)$ and $M(\cdot)$ that appear in the axioms (ii) and (iii) are given, respectively, by $H=1, K(t)=1$ and $M(t)=e^{-\beta t}$, for all $t \geq 0$.

Taking into account what was said before we derive the following result.

Theorem 3.1. Assume that all previous conditions are fulfilled. Assume in addition that the following inequalities hold,

$$
\int_{\frac{3\|\phi\|}{1-\sum_{i=1}^{m} L_{i}}}^{b} p(s) \mathrm{d} s<\infty,
$$

where $p(\cdot)$ represents the right-hand side of the inequalities (3.4). Then the problem (3.2) has a mild solution.

\section{Competing interests}

The authors declare that they have no competing interests.

\section{Authors' contributions}

MNR conceived the study and participated in its design and coordination. MH participated in the design of the study and performed the typesetting of the text. GS participated in the design of the article. All authors read and approved the final manuscript.

\footnotetext{
Author details

${ }^{1}$ Departamento De Matemática, Universidade Federal De Pernambuco, Recife-pe, Cep 50540-740, Brazil ${ }^{2}$ Faculdade De Ciência E Tecnologia De Caruaru, Universidade De Pernambuco, Caruaru-pe, Cep 55002, Brazil

Received: 1 March 2011 Accepted: 7 December 2011 Published: 7 December 2011

References

1. Bainov, DD, Pavel, S: Impulsive Differential Equations: Periodic Solutions and Applications. In: Pitman Monographs and Surveys in Pure and Applied Mathematics. pp. x+228. Longman Scientific \& Technical, Harlow, UK66, (1993)

2. Samoilenko, AM, Perestyuk, NA: Impulsive Differential Equations. World Scientific, Singapore (1995)

3. Lakshmikanthan, V, Bainov, D, Simeonov, P: Theory of Impulsive Differential Equations. In series in Modern Applied Mathematics. World Scientific, Teaneck, NJ. 6 (1989)
} 
4. Park, JY, Balachandran, K, Annapoorani, N: Existence results for impulsive neutral functional integrodifferential equations with infinite delay. Nonlinear Anal. 71, 3152-3162 (2009). doi:10.1016/j.na.2009.01.192

5. Balachandran, K, Annapoorani, N: Existence results for impulsive neutral evolution integrodifferential equations with infinite delay. Nonlinear Anal Hybrid Syst. 3, 674-684 (2009). doi:10.1016/.j.nahs.2009.06.004

6. Schnaubelt, R: Asymptotic behavior of parabolic nonautonomous evolution equations. In Functional Analytic Methods for Evolution Equations. Lecture Notes in Mathematics, vol. 1855, pp. 401-472.Springer, Berlin (2004)

7. Hernández, E, Prokopezyk, A, Ladeira, L: A note on partial functional differential equations with state-dependent delay. Nonlinear Anal Real World Appl. 7(4), 510-519 (2006). doi:10.1016/j.nonrwa.2005.03.014

8. Baghli, S, Benchohra, M: Perturbed functional and neutral functional evolution equations with infinite delay in Fréchet spaces. Elec J Differ Equ. 69, 1-19 (2008)

9. Ahmad, S, Stamov, GT: Almost periodic solutions of $\mathrm{N}$-dimensional impulsive competitive systems. Nonlinear Anal Real World Appl. 10(3), 1846-1853 (2009). doi:10.1016/..nonrwa.2008.02.020

10. Ahmad, S, Stamov, GT: On almost periodic processes in impulsive competitive with delay and impulsive perturbations. Nonlinear Anal Real World Appl. 10(5), 2857-2863 (2009). doi:10.1016/j.nonrwa.2008.09.003

11. Hernádez, $\mathrm{E}$, Henráquez, $\mathrm{H}$ : Existence of periodic solutions of partial neutral functional differential equations with unbounded delay. J Math Anal Appl. 221(1), 499-522 (1998)

12. Herniández, E, Pierri, M, Gonáalves, G: Existence results for an impulsive abstract partial differential equation with statedependent delay. Comput Math Appl. 52(3-4), 411-420 (2006). doi:10.1016/j.camwa.2006.03.022

13. Hernández, E, Rabelo, M, Henráquez, H: Existence of solutions for impulsive partial neutral functional differential equations. J Math Anal Appl. 331, 1135-1158 (2007). doi:10.1016/j.jmaa.2006.09.043

14. Chang, YK, Anguraj, A, Arjunan, MM: Existence results for impulsive neutral functional differential equations with infinite delay. Nonlinear Anal Hybrid Syst. 2, 209-218 (2008). doi:10.1016/j.nahs.2007.10.001

15. Cuevas, C, Hernández, E, Rabelo, M: The existence of solutions for impulsive neutral functional differential equations. Comput Math Appl. 58(4), 744-757 (2009). doi:10.1016/j.camwa.2009.04.008

16. Hernández, E, Hernráquez, HR: Impulsive partial neutral differential equations. Appl Math Lett. 19, 215-222 (2006). doi:10.1016/j.aml.2005.04.005

17. Hernádez, E, McKibben, M, Henráquez, HR: Existence results for abstract impulsive second order neutral functional differential equations. Nonlinear Anal Theory Methods Appl. 70(7), 2736-2751 (2009). doi:10.1016/j.na.2008.03.062

18. Stamov, GT: On the existence of almost periodic solutions for the impulsive Lasota-Wazewska model. Appl Math Lett. 22(4), 516-520 (2009). doi:10.1016/j.aml.2008.07.002

19. Stamov, GT: Almost periodic models of impulsive Hopfield neural networks. J Math Kyoto Univ. 49(1), 56-67 (2009)

20. Stamov, GT: Stability processes of moving invariant manifolds in uncertain impulsive differential-difference equations. Math Bohem. 134(1), 67-76 (2009)

21. Stamov, GT: Existence of almost periodic solutions for impulsive cellular neural networks. Rocky Mountain J Math. 38(4), 1271-1284 (2008). doi:10.1216/RMJ-2008-38-4-1271

22. Benchohra, M, Slimani, BA: Existence and uniqueness of solutions to impulsive fractional differential equations. Elec J Differ Equ. 2009(10), 11 (2009)

23. Agarwal, RP, Benchohra, M, Slimani, BA: Existence results for differential equations with fractional order and impulses. Mem Diff Equ Math Phys. 44, 1-21 (2008)

24. Vrabie, II: $C_{0}$-semigroups and applications. North-Holland Mathematics Studies. pp. xii+373. North-Holland Publishing Co., Amsterdam191, (2003)

25. Pazy, A: Semigroups of linear operators and applications to partial differential equations. In Applied Mathematical Sciences, vol. 44,Springer, New York (1983)

26. Lunardi, A: Analytic Semigroups and Optimal Regularity in Parabolic Problems. In Progress in Nonlinear Differential Equations and Their Applications, vol. 16,Birkháauser Verlag, Basel (1995)

27. Granas, A, Dugundji, J: Fixed Point Theory. Springer, New York (2003)

28. Martin, RH Jr: Nonlinear Operators and Differential Equations in Banach Spaces. Pure and Applied Mathematics. pp. xi +440.Wiley, New York (1976)

29. Hino, Y, Murakami, S, Naito, T: Functional-Differential Equations with Infinite Delay. Lectures Notes in Mathematics. Spring, New York1473 (2003)

doi:10.1186/1029-242X-2011-135

Cite this article as: Rabelo et al:: Existence of integro-differential solutions for a class of abstract partial impulsive differential equations. Journal of Inequalities and Applications 2011 2011:135.

\section{Submit your manuscript to a SpringerOpen ${ }^{\circ}$ journal and benefit from:}

- Convenient online submission

- Rigorous peer review

- Immediate publication on acceptance

- Open access: articles freely available online

- High visibility within the field

- Retaining the copyright to your article

Submit your next manuscript at $\boldsymbol{s p r i n g e r o p e n . c o m ~}$ 